\title{
Stickstoffmonoxid in verschiedenen Kompartimenten des Atemtrakts - Vergleich der Daten von Nichtrauchern und Rauchern
}

\author{
Bronchial and Alveolar NO Parameters in Smokers
}

Autoren

Institut

\section{Barbinova, A. Preisser, X. Baur}

Ordinariat und Zentralinstitut für Arbeitsmedizin und Maritime Medizin (ZfAM), University Medical Center Hamburg-Eppendorf eingereicht 1.4 .2010

akzeptiert nach Revision 11. 10.2010

\section{Bibliografie}

Dol http://dx.doi.org/ 10.1055/s-0030-1255886 Online-Publikation: 26.11.2010

Pneumologie 2011; 65:

103-109 @ Georg Thieme

Verlag KG Stuttgart · New York ISSN 0934-8387

\section{Korrespondenzadresse}

Liubov Barbinova

Ordinariat und Zentralinstitut

für Arbeitsmedizin und

Maritime Medizin Hamburg

Seewartenstraße 10

20459 Hamburg

lioukov@uke.uni-hamburg.de

\section{Zusammenfassung \\ $\nabla$}

Wir untersuchten mittels verschiedener Atemflüsse, ob sich Unterschiede zwischen Rauchern und Nichtrauchern hinsichtlich der differenziellen NO-Parameter Bronchialwand-Konzentration $\left(C_{a w}\right)$, alveolare Konzentration $\left(C_{\text {alv }}\right)$ und Diffusionskoeffizient $\left(D_{a w n o}\right)$ ergeben. Die 34 untersuchten beschwerdefreien, nicht therapierten Raucher weisen im Vergleich zu 43 gesunden Nichtrauchern signifikant niedrigere FeNO-Werte auf. Die Analyse der differenziellen NO-Parameter mittels des 2-Kompartimenten-Modells der NOProduktion belegt unter Rauchern eine signifikant verminderte bronchiale NO-Konzentration, jedoch keine signifikante Änderung der dem Alveolarbereich zugerechneten NO-Fraktion. Die Zusammenhänge zwischen den einzelnen NO-Parametern der Nichtraucher passen zum 2-Kompartimenten-Modell. Im Gegensatz dazu weisen Raucher eine monotone positive Korrelation zwischen den verminderten $C_{a w}$ und FeNO unter allen fünf Flussraten auf. Das könnte darauf zurückzuführen sein, dass das alveolare NO der Raucher teilweise aus dem bronchialen Bereich stammt. Das passt zu deren signifikant erniedrigten $\mathrm{FEV}_{1^{-}}$, $\mathrm{FEF}_{50^{-}}$und $\mathrm{FEF}_{75^{-}}$-Werten, welche mit Turbulenzen und unterschiedlichen Verzögerungen des exspiratorischen Gasabflusses im Bronchiolo-Alveolarbereich einhergehen. Wir stellen die Hypothese auf, dass der verminderten NOKonzentration in der Bronchialwand des Rauchers eine pathophysiologische Rolle bei der Manifestation eines Small airways disease zukommt.

\section{Einleitung}

\section{$\nabla$}

Die Bestimmung des fraktionierten Stickstoffmonoxids in der Ausatemluft (FeNO) ist ein relativ neues, wenig belastendes und leicht durchführbares Verfahren, das zunehmend in pneumologischen Zentren eingesetzt wird. In einer Viel-

\section{Abstract \\ $\nabla$}

Our aim was to determine by means of five exhaled flow rates differential parameters of FeNO and the relations between them in smokers and non-smokers. 34 smokers (without respiratory symptoms and medication) were examined. Compared to 43 healthy non-smokers, FeNO was significantly lower. The analysis of the differential NO parameters by means of the two-compartment model of NO production revealed a significant decrease in the bronchial NO concentration, but no significant changes of $\mathrm{NO}$ in alveolar fraction by smokers. The relations between differential parameters in non-smokers confirm the theoretical expectations of the two-compartment model. Conversely, smokers exhibit an abnormal remarkable correlation, namely, a high correlation between the reduced $C_{a w}$ und FeNO at all five flow rates. It may be assumed that the alveolar NO fraction in smokers is to some extent of bronchial origin. Such a preposition is in line with significantly decreases of $\mathrm{FEV}_{1}, \mathrm{FEF}_{50}$ and $\mathrm{FEF}_{75}$ in smokers, which are associated with turbulences, flow inhomogeneity and a varied delay of expiration time from the peripheral airways. We hypothesize that the decreased NO concentration in the bronchial wall of smokers plays a pathophysiological role in the genesis of small airways disease. 
Monitoring sich zur Vorhersage von Exazerbationen und Rückfällen eignet und dabei sogar der Lungenfunktionsverlaufskontrolle überlegen sein kann [5 - 7]. Allerdings gibt es vereinzelt auch entgegengesetzte Ergebnisse [8].

FeNO stellt einen integrativen Parameter dar, wobei bisher weitgehend unklar ist, ob die einzelnen Abschnitte des Respirationstrakts bei verschiedenen Atemwegs- und Lungenkrankheiten Abweichungen der NO-Konzentration aufweisen.

Ein in Anlehnung an das grundlegende Konzept von Tsoukias et al. [9] entwickeltes 2-Kompartimenten-Modell erlaubt die Ermittlung der NO-Konzentration in den verschiedenen Abschnitten des ganzen Respirationstrakts, nämlich im alveolaren $\left(\mathrm{C}_{\text {alv }}\right)$ und bronchialen Bereich $\left(\mathrm{C}_{\mathrm{aw}}\right)$, ferner die Bestimmung der NODiffusionskapazität der Bronchialwand $\left(\mathrm{D}_{\mathrm{awN}}\right)$. Das Modell ist einfach und betrachtet das bronchiale System als ein Rohr mit einem konstanten Radius und mit konstanter NO-Konzentration in der Bronchialwand und den Alveolarbereich als ein Reservoir mit konstanter und einheitlicher NO-Konzentration.

Dem bronchialen Kompartiment entsprechen die ersten 16 Generationen der Bronchien (konduktive Zone). Zum Alveolarbereich gehören nicht nur die Alveolen, sondern auch die alveolisierten Bronchiolen der 17. -23. Generationen (Gasaustauschbereich). Die Diffusionskapazität wird als $D_{a w n o}=\lambda \times A$ definiert, wobei A die totale Fläche der bronchialen konduktiven Zone und $\lambda$ der Transfer-Koeffizient des NO pro der Flächeneinheit sind.

Von theoretisch-konstruktiver Seite basiert das Modell auf der Gasdiffusion in einem Rohr; sein mathematisches Konzept lehnt sich an die generelle Form des Transport-Modells an $[9,10]$. Es beschreibt die Änderung der ausgeatmeten NO-Konzentration (FeNO) und den entsprechenden NO-Strom aus der gesamten Lunge als Funktion der drei vorgenannten Parameter und der Flussrate während der Ausatmung. Obwohl das 2-Kompartimenten-Modell sehr einfach ist, erklärt es gut praktische Gesetzmäßigkeiten der NO-Dynamik im gesamten Respirationstrakt [11], so die Abhängigkeit des FeNO und der NO-Abgabemenge (pro Luftvolumeneinheit der bronchialen Wand) von dem exhalierten Fluss: FeNO sinkt (abgeflachter hyperbolischer Verlauf) mit zunehmender Flussrate $\left(V^{\prime}\right)(\boldsymbol{A}$ Abb. 1 a); der NO-Strom (NO-Produktion) nimmt demgegenüber nahezu linear parallel mit $V^{\prime}$ zu (৫ Abb. 1b).

Unter Anwendung verschiedener Flussraten, d.h. unterschiedlicher Passagezeiten und damit einer unterschiedlich großen NO-Abgabemenge aus den bronchialen Wänden, lassen sich mit Hilfe exponenzieller Gleichungen (oder deren linearer Approximation) die drei Kenngrößen $C_{a w}, C_{\text {alv }}$ und $D_{\text {awNo }}$ erfassen:

$C_{e x}=C_{a w} \times\left(1-\exp \left(-D_{a w n o} / V^{\prime}\right)\right)+C_{a l v} \times \exp \left(-D_{a w n o} / V^{\prime}\right)$

(wobei $\mathrm{C}_{\mathrm{ex}}$ die gemessene Konzentration des exhalierten NO in ppb unter dem jeweiligen $\mathrm{V}^{\prime}$ in $\mathrm{mL} \times \mathrm{s}^{-1}$ dargestellt).

Unser Ziel war, die differenziellen NO-Parameter zu ermitteln und hierbei zu untersuchen, ob zwischen Rauchern und Nichtrauchern Unterschiede bestehen.

Wir überprüften auch, ob die berechneten Parameter zu den theoretischen Modell-Voraussetzungen passen.

\section{Methoden}

$\nabla$

Die demografischen und klinischen Daten der 77 freiwilligen Probanden (43 Nichtraucher und 34 Raucher), die nach Fragebogenangaben alle keine respiratorischen Erkrankungen aufwiesen, sind in $\bigcirc$ Tab. 1 dargestellt. Raucher wurden gebeten, mindestens 2 Stunden vor der Untersuchung nicht zu rauchen. Keiner

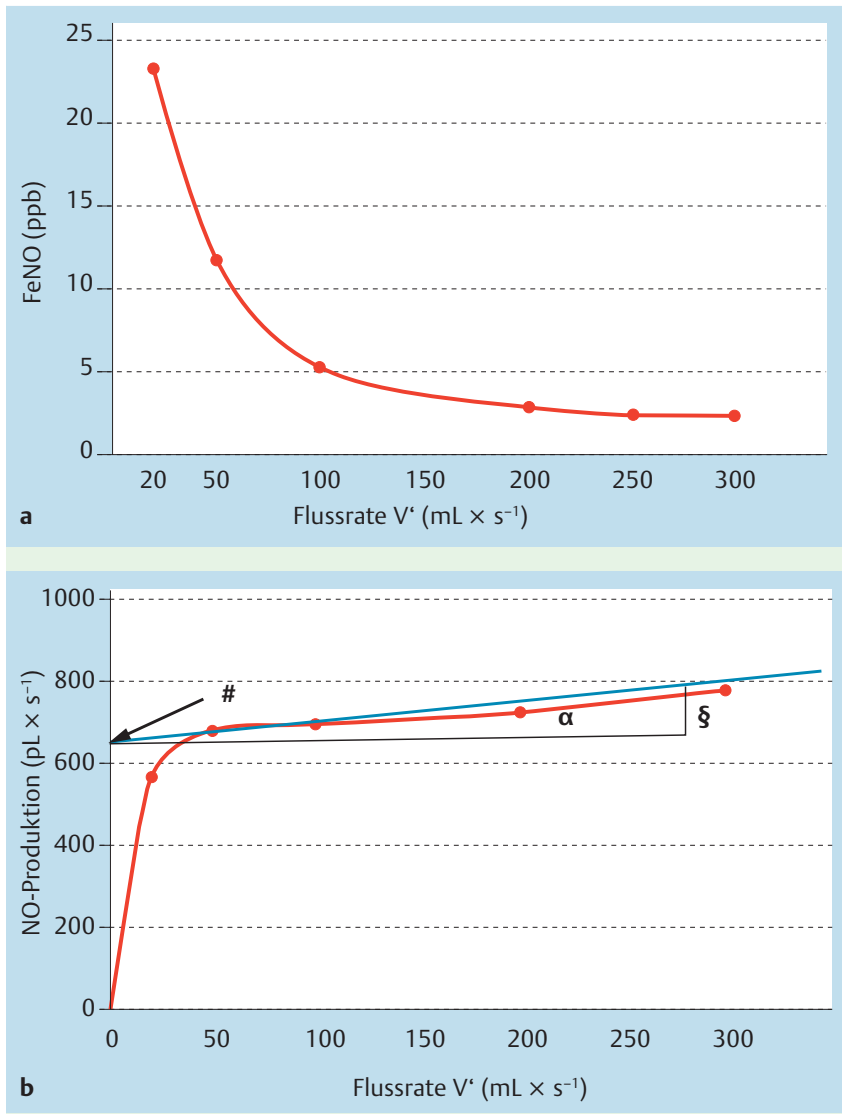

Abb. 1 a FeNO (in ppb) als Funktion der exhalierten Flussrate V'. Die Kurve präsentiert die Mittelwerte der aktuellen Studie $(\mathrm{N}=77)$. b NO-Strom (geschätzt als $C_{\mathrm{ex}} \times \mathrm{V}^{\prime}$ ) unter verschiedenen Flussraten. \# dieser Wert auf der $y$-Achse entspricht annähernd dem maximalen bronchialen Strom ( $\left.\mathrm{J}_{\mathrm{aw}}=\mathrm{C}_{\mathrm{aw}} \times \mathrm{D}_{\mathrm{awN}}\right)$ ), $\S$ tan $\alpha$ entspricht annähernd der alveolaren Konzentration $C_{\text {alv }}$ (modifiziert nach [12]). Die Grafik präsentiert ebenfalls die aktuellen Daten (Mittelwerte des gesamten Kollektivs).

Tab. 1 Demografische und klinische Daten der untersuchten Kollektive.

\begin{tabular}{|c|c|c|}
\hline & $\begin{array}{l}\text { Nichtraucher } \\
(\mathrm{N}=43)\end{array}$ & $\begin{array}{l}\text { Raucher } \\
(N=34)\end{array}$ \\
\hline Alter, Median (Range) & $46(16-64)$ & $48(23-78)$ \\
\hline Geschlecht, w/m & $17 / 26$ & $12 / 22$ \\
\hline $\mathrm{FEV}_{1}, \%$ Soll & $96,9 \pm 2,2$ & $87,0 \pm 3,6^{*}$ \\
\hline $\mathrm{FEV}_{1} / \mathrm{FVC}, \%$ & $76,5 \pm 0,9$ & $74,4 \pm 1,9$ \\
\hline FVC, \% Soll & $97,4 \pm 2,4$ & $93,0 \pm 3,5$ \\
\hline $\mathrm{FEF}_{25}, \%$ Soll & $96,1 \pm 4,1$ & $83,6 \pm 4,5(p=0,07)$ \\
\hline $\mathrm{FEF}_{50}, \%$ Soll & $90,1 \pm 4,3$ & $71,8 \pm 5,0 *$ \\
\hline $\mathrm{FEF}_{75}, \%$ Soll & $85,26 \pm 4,7$ & $63,9 \pm 4,7^{* *}$ \\
\hline
\end{tabular}

der Probanden nahm Kortikosteroide und/oder $\beta_{2}$-Sympathomimetika. Bei allen Probanden wurden die spirometrischen Lungenfunktionsparameter bestimmt.

Die NO-Analysen wurden entsprechend den Empfehlungen von ATS/ERS [13] mit dem Chemilumineszenzanalysator ECO-Physics-88 (ECO-Physics, Schweiz) durchgeführt. Die Messung erfolgte während einer langsamen Ausatmung gegen einen Druck von ca. $10 \mathrm{~cm} \mathrm{H}_{2} \mathrm{O}$. 


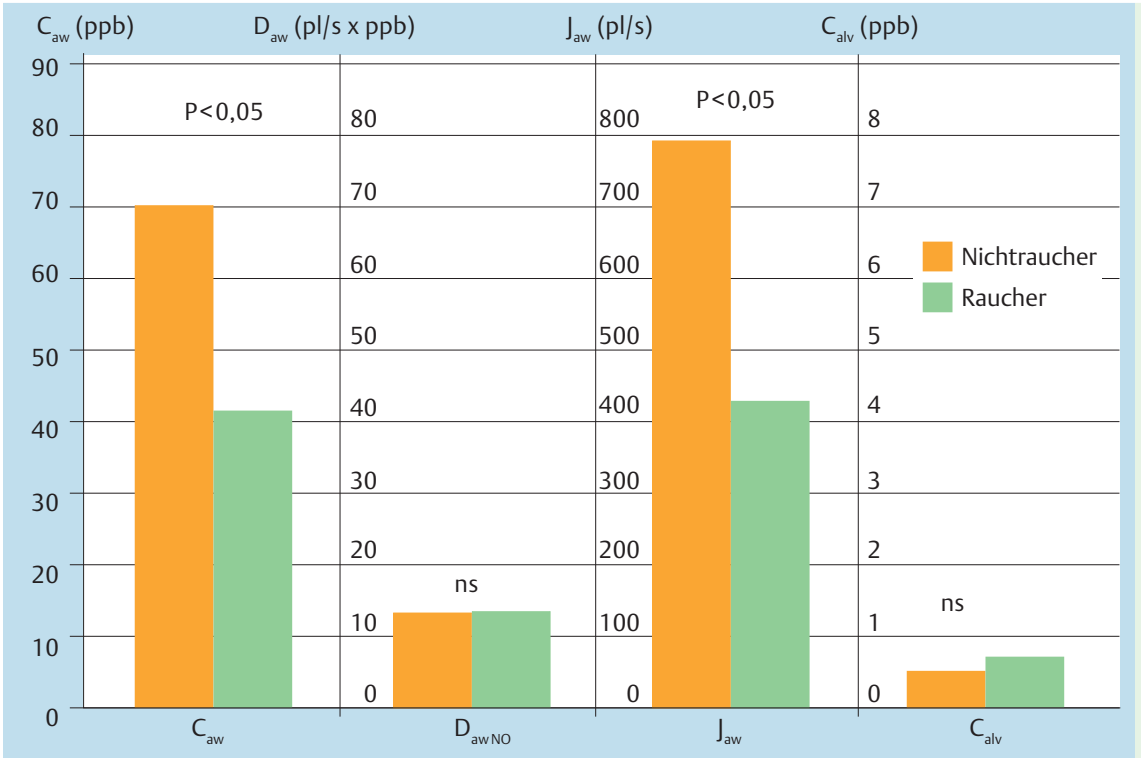

Abb. 2 Differenzielle FeNO-Werte von 38 Nichtrauchern und 29 Rauchern.

Jaw und $C_{a w}$ sind bei Rauchern signifikant niedriger als bei Nichtrauchern $(427,6 \pm 53,9$ vs. 789,0 $\pm 57,5 \mathrm{pl} \times \mathrm{s}^{-1}, \mathrm{p}<0,001$ bzw. $41,8 \pm 6,4$ vs. 69,4 $\pm 5,0 \mathrm{ppb}, \mathrm{p}<0,005)$. $\mathrm{C}_{\mathrm{alv}}$ und $\mathrm{D}_{\text {awno }}$ der Raucher unterscheiden sich nicht von jenen der Nichtraucher $(0,7 \pm 0,1$ ppb vs. $0,5 \pm 0,1$ ppb bzw. $13,5 \pm 1,6$ ppb vs. $13,1 \pm 1,2 \mathrm{pl} / \mathrm{s} \times \mathrm{ppb}$; beide $\mathrm{ns})$.

Den FeNO-Basalwert bestimmten wir standardisiert nach den ATS/ERS-Empfehlungen bei einem Atemfluss von $50 \mathrm{~mL} / \mathrm{s}$ (FeNO $=\mathrm{FeNO}_{50}$ ).

Wir führten die differenzierende FeNO-Messung unter Verwendung von 5 verschiedenen Flussraten $(20,50,100,200$, $300 \mathrm{~mL} / \mathrm{s}$ ) durch, wobei jeweils mindestens 2-fach-Messungen vorgenommen wurden. Für jede Messung wurde das Verhältnis zwischen der exhalierten Konzentration und V' mittels der Gleichung (1) modelliert. Dies ergab für jeden Probanden ein System aus 10-15 Datensätzen. Des Weiteren schätzten wir mittels des nichtlinearen Regressionsprogramms (SAS 8.1; Newtons Algorithmus) die Parameter $C_{a l v}, C_{a w}$ und $D_{a w N o}$ für jeden Probanden. Die Prozedur der Schätzung wird hierbei erfolgreich erfüllt (Konvergenz), wenn man mittels eines schrittweisen Verfahrens (Iteration) zu der Lösung kommt, die das Minimum der Fehlerquadratsumme liefert.

\section{Ergebnisse}

\section{Lungenfunktionswerte}

$\mathrm{FEV}_{1}, \mathrm{FEF}_{50}$ und $\mathrm{FEF}_{75}$ sind unter Rauchern signifikant niedriger als unter Nichtrauchern. Die anderen Lungenfunktionsparameter zeigen keine wesentlichen Unterschiede ( Tab. 1).

\section{FeNO-Werte und differenzielle NO-Parameter von Nichtrauchern und Rauchern}

Der FeNO-Mittelwert des Nichtraucher-Kollektivs beträgt 13,7 $\pm 0,9 \mathrm{ppb}$. Dies stimmt gut mit unserer früheren Studie [14] überein, in der Referenzwerte von gesunden Personen ermittelt wurden (15,4 $\pm 1,1 \mathrm{ppb} ; \mathrm{n}=37)$.

37 von 43 nichtrauchenden Probanden (86\%) haben ein normales basales FeNO (unterhalb des oberen Grenzwertes von 20 ppb); 6 weisen leicht erhöhte Werte (im Bereich 20-30 ppb) auf.

Von den 34 rauchenden Probanden zeigt nur einer (ein Atopiker) einen erhöhten Wert (23,8 ppb). Der Mittelwert des Kollektivs der Raucher beträgt 7,9 $\pm 1,1 \mathrm{ppb}$. Der Unterschied des $\mathrm{FeNO}_{50}$ zwischen Nichtrauchern und Rauchern ist signifikant ( $<<0,001$ ). Die Analyse der NO-Werte unter anderen Flüssen ergibt auch sig-
Tab. 2 Vergleich der FeNO-Werte unter verschiedenen Flüssen.

\begin{tabular}{|c|c|c|}
\hline & Nichtraucher & Raucher \\
\hline $\mathrm{FeNO}_{20}$ & $27,9 \pm 2,0$ & $16,7 \pm 1,8^{* * *}$ \\
\hline $\mathrm{FeNO}_{50}$ & $13,7 \pm 0,9$ & $7,9 \pm 0,8^{* * *}$ \\
\hline $\mathrm{FeNO}_{100}$ & $7,1 \pm 0,5$ & $4,7 \pm 0,5^{* *}$ \\
\hline $\mathrm{FeNO}_{200}$ & $4,3 \pm 0,4$ & $2,7 \pm 0,3^{* *}$ \\
\hline $\mathrm{FeNO}_{300}$ & $2,9 \pm 0,2$ & $2,0 \pm 0,2^{*}$ \\
\hline
\end{tabular}

Alle Werte sind als MW \pm SEM dargestellt. ${ }^{*} p<0,05,{ }^{* *} p<0,005$,

${ }^{* * *} p<0,0005$ im Vergleich zum Nichtraucher-Kollektiv.

nifikante Unterschiede mit höheren Werten unter Nichtrauchern (๑ Tab.2).

Wir schätzten schrittweise mittels nichtlinearer Regression die gesuchten Parameter $C_{a w}, C_{a l v}$ und $D_{a w n o}$. Die Prozedur der Schätzung wurde erfolgreich bei 67 der 77 Patienten (38 Nichtraucher, 29 Raucher) erfüllt, bei 10 Patienten gelang dies nicht. Die FeNOKonzentrationen der 67 Probanden fallen ähnlich aus wie jene des Gesamtkollektivs (NR: 13,6 $\pm 0,9 \mathrm{ppb} ; \mathrm{R}: 8,2 \pm 1,0 \mathrm{ppb}$; $\mathrm{p}<0,005)$. Der Vergleich der klinischen und spirometrischen Daten zwischen den ursprünglich 77 Probanden und den 67 Patienten mit berechenbaren differenziellen Parametern ergibt keine Unterschiede.

Zwei der vier berechneten flussunabhängigen Parameter, $\mathrm{J}_{\mathrm{aw}}$ und $\mathrm{C}_{\mathrm{aw}}$, weisen zwischen Rauchern $(\mathrm{n}=29)$ und Nichtrauchern $\left(\mathrm{n}=38\right.$ ) signifikante Abweichungen auf. $\mathrm{C}_{\mathrm{alv}}$ und $\mathrm{D}_{\text {awNo }}$ zeigen keine signifikanten Unterschiede $(\bullet$ Abb. 2).

Wir fanden keine Korrelation zwischen den Lungenfunktionsparametern und den differenziellen NO-Parametern der Nichtraucher. Für Raucher ergab sich eine positive Korrelation zwischen $\mathrm{FEV}_{1} / \mathrm{FVC}$ und $\mathrm{C}_{\mathrm{aw}}\left(\mathrm{r}_{\mathrm{sp}}=0,38 ; \mathrm{p}<0,05\right)$.

\section{Validierung des Modells und Analyse der einzelnen Parameter}

Wir verglichen beide Kollektive bezüglich der Korrelationen zwischen den exhalierten NO-Konzentrationen unter verschiedenen Flüssen $\left(\mathrm{FeNO}_{20-300}\right)$ einerseits und den differenziellen, nach dem 2-Kompartimenten-Modell berechneten NO-Parametern andererseits ( $\bullet$ Tab.3). 
Tab. 3 Korrelationen zwischen $\mathrm{FeNO}_{20-300}$ und den einzelnen differenziellen NO-Parametern. Die Abweichungen zwischen beiden Kollektiven bzgl. der Korrelationen sind in Kursivschrift wiedergegeben.

\begin{tabular}{|c|c|c|c|c|}
\hline \multicolumn{5}{|c|}{ a. Korrelationen bei Nichtrauchern } \\
\hline & $\mathrm{C}_{\mathrm{alv}}$ & $\mathrm{C}_{\mathrm{aw}}$ & Dawno $_{\text {aw }}$ & Jaw \\
\hline $\mathrm{FeNO}_{20}$ & keine & $\begin{array}{l}r_{s p}=0,37 ; \\
p<0,05\end{array}$ & keine & $\begin{array}{l}r_{s p}=0,84 \\
p<0,0001\end{array}$ \\
\hline $\mathrm{FeNO}_{50}$ & keine & keine & $\begin{array}{l}r_{s p}=0,44 \\
p<0,05\end{array}$ & $\begin{array}{l}r_{s p}=0,92 ; \\
p<0,0001\end{array}$ \\
\hline $\mathrm{FeNO}_{100}$ & keine & keine & $\begin{array}{l}r_{s p}=0,36 \\
p<0,05\end{array}$ & $\begin{array}{l}r_{s p}=0,90 \\
p<0,0001\end{array}$ \\
\hline $\mathrm{FeNO}_{200}$ & keine & keine & $\begin{array}{l}r_{s p}=0,32 ; \\
p<0,05\end{array}$ & $\begin{array}{l}r_{s p}=0,95 \\
p<0,0001\end{array}$ \\
\hline $\mathrm{FeNO}_{300}$ & $\begin{array}{l}r_{s p}=0,38 \\
p<0,05\end{array}$ & keine & $\begin{array}{l}r_{s p}=0,37 \\
p<0,05\end{array}$ & $\begin{array}{l}r_{s p}=0,87 ; \\
p<0,0001\end{array}$ \\
\hline \multicolumn{5}{|c|}{ b. Korrelationen bei Rauchern } \\
\hline & $\mathrm{C}_{\text {alv }}$ & $\mathrm{C}_{\mathrm{aw}}$ & Dawno $_{\text {aw }}$ & Jaw \\
\hline $\mathrm{FeNO}_{20}$ & keine & $\begin{array}{l}r_{s p}=0,87 ; \\
p<0,0001\end{array}$ & keine & $\begin{array}{l}r_{s p}=0,93 ; \\
p<0,0001\end{array}$ \\
\hline $\mathrm{FeNO}_{50}$ & keine & $\begin{array}{l}r_{s p}=0,81 ; \\
p<0,0001\end{array}$ & keine & $\begin{array}{l}r_{s p}=0,97 ; \\
p<0,0001\end{array}$ \\
\hline $\mathrm{FeNO}_{100}$ & keine & $\begin{array}{l}r_{s p}=0,78 \\
p<0,0001\end{array}$ & keine & $\begin{array}{l}r_{s p}=0,96 \\
p<0,0001\end{array}$ \\
\hline $\mathrm{FeNO}_{200}$ & $\begin{array}{l}r_{s p}=0,38 \\
p<0,05\end{array}$ & $\begin{array}{l}r_{s p}=0,83 \\
p<0,0001\end{array}$ & keine & $\begin{array}{l}r_{s p}=0,93 ; \\
p<0,0001\end{array}$ \\
\hline $\mathrm{FeNO}_{300}$ & $\begin{array}{l}r_{s p}=0,48 ; \\
p<0,01\end{array}$ & $\begin{array}{l}r_{s p}=0,82 ; \\
p<0,0001\end{array}$ & keine & $\begin{array}{l}r_{s p}=0,87 \\
p<0,0001\end{array}$ \\
\hline
\end{tabular}

$\mathrm{FeNO}_{20}$ bis $\mathrm{FeNO}_{300}$ (FeNO bei angewandten Flüssen) zeigen in beiden Kollektiven eine enge positive Korrelation mit dem NOStrom aus der bronchialen Wand $\left(\mathrm{J}_{\mathrm{aw}}\right)\left(\mathrm{r}_{\mathrm{Sp}}=0,87-0,97\right)$.

Auch die Korrelation zwischen $\mathrm{C}_{\mathrm{alv}}$ und $\mathrm{FeNO}_{20-300}$ ist in beiden Kollektiven ähnlich: Es besteht keine Korrelation unter den niedrigen Flüssen, jedoch eine signifikante Korrelation für die Flüsse $\mathrm{FeNO}_{200}$ und $\mathrm{FeNO}_{300}$.

$\mathrm{C}_{\mathrm{aw}}$ und $\mathrm{D}_{\mathrm{awNO}}$ weisen deutliche Unterschiede zwischen den beiden Kollektiven auf.

Nichtraucher zeigen eine positive Korrelation von $C_{a w}$ mit der exhalierten NO-Konzentration unter der niedrigsten Flussrate $\left(\mathrm{FeNO}_{20}\right)$ und keinen derartigen Zusammenhang unter höheren Flussraten. $\mathrm{D}_{\mathrm{awNO}}$, die negativ mit $\mathrm{C}_{\mathrm{aw}}$ korreliert, weist unter Nichtrauchern (nicht aber unter Rauchern) signifikante Korrelationen mit $\mathrm{FeNO}_{50-300}$ auf.

Insgesamt zeigen sowohl die bronchialen als auch die alveolaren NO-Konzentrationen $\left(\mathrm{C}_{\mathrm{aw}}, \mathrm{C}_{\mathrm{alv}}\right)$ mit $\mathrm{FeNO}_{20-300}$ im NichtraucherKollektiv Zusammenhänge, die prinzipiell zum 2-Kompartimenten-Modell passen: Unter höheren Flüssen kommt das exhalierte NO zu einem Großteil direkt aus dem alveolaren Bereich und nur zu einem geringeren Teil aus den Bronchien (aufgrund der kurzen Passagezeit). Entsprechend nähert sich hier die ausgeatmete NO-Konzentration $C_{\mathrm{ex}}$ der alveolaren Konzentration $\mathrm{C}_{\mathrm{alv}}$. Während langsamer Ausatmung (unter niedriger Flussrate) diffundiert pro Einheit Atemluft mehr NO aus der Bronchialwand; entsprechend nähert sich die ausgeatmete NO-Konzentration $\mathrm{C}_{\mathrm{ex}}$ der Konzentration im bronchialen Bereich $\mathrm{C}_{\mathrm{aw}}$. Die Korrelationsverhältnisse in $\bullet$ Tab. 3 a befinden sich in Übereinstimmung mit diesen theoretischen Erwartungen des 2-KompartimentenModells (vgl. auch Abb.1a). Auch die positive Korrelation zwischen FeNO und $\mathrm{D}_{\text {awNo }}$ unter mittleren Flüssen passt grundsätzlich zu den strukturellen und physiologischen Annahmen des 2-Kompartimenten-Modells. $\mathrm{D}_{\text {awno }}$ ist proportional der Fläche der bronchialen konduktiven Zone (A) sowie dem Bronchienvolumen $(\mathrm{V})$ und der Passagezeit, wobei Letztere den limi- tierenden Faktor der Diffusion aus der Bronchialwand darstellt. Der FeNO-Verlauf zwischen niedrigen Flüssen (wo FeNO sich $C_{a w}$ nähert) und hohen Flüssen (wo FeNO sich $C_{\text {alv }}$ nähert) wird von $\mathrm{D}_{\text {awNo }}$ bestimmt ( Abb. 1 a). Dieser Aspekt wurde von theoretischer Seite in der analytischen Arbeit von Jörres [15] betrachtet. Bei kleinen $\mathrm{D}_{\mathrm{awNO}}$-Werten fällt die Kurve steil $\mathrm{ab}$, bei höheren $\mathrm{D}_{\mathrm{awnO}}$-Werten flacht sie ab. Die von uns gefundenen Korrelationen bestätigen diese Konstellationen von der experimentellpraktischen Seite; es ergibt sich ein positiver Zusammenhang unter mittleren Flussraten: Je größer $\mathrm{D}_{\mathrm{awNo}}$ ist, desto größer ist FeNO.

Im Gegensatz zu Nichtrauchern ergibt sich für Raucher eine monotone positive Korrelation zwischen $C_{a w}$ und FeNO unter allen fünf Flussraten. Es sieht so aus, als ob unter Rauchern FeNO nur von $C_{a w}$ (auch bei hohen Flussraten) bestimmt wird. Entsprechend ist hier $C_{e x}$ immer mit $C_{a w}$ korreliert, unabhängig davon, welche Flussraten vorliegen. Ein solches Verhältnis passt nicht zum 2-Kompartimenten-Modell und kann durch Turbulenzen und Inhomogenitäten im alveolo-bronchiolaren Bereich der Raucher erklärt werden. Dies zeigt letztendlich auch die Grenze des 2-Kompartimenten-Modells, welches $\mathrm{C}_{\mathrm{aw}}$ und $\mathrm{C}_{\mathrm{alv}}$ als unabhängige Komponenten betrachtet.

\section{Diskussion und Schlussfolgerungen}

Die sowohl im Nichtraucher- als auch im Raucher-Kollektiv unter verschiedenen Flüssen festzustellende stark positive Korrelation zwischen FeNO und dem NO-Strom aus der Bronchialwand Jaw bedeutet, dass $\mathrm{J}_{\mathrm{aw}}$ keine zusätzliche Information liefert, wenn wir uns nur für die bronchiale NO-Produktion als Ganzes interessieren.

Für $C_{a l v}, C_{a w}$ und $D_{a w N o}$ ergibt sich keine solche direkte lineare Abhängigkeit von FeNO. Dies erlaubt hinsichtlich lokalisierter Prozesse zusätzliche Aussagen in verschiedenen Kollektiven und im Einzelfall.

\section{Hinweise auf eine Vermischung des alveolaren und bronchialen NO bei Rauchern bzw. COPD}

In unserer Studie weisen Raucher bei signifikant erniedrigter $C_{a w}$ auffallende Korrelationen zwischen diesem differenziellen NOParameter und FeNO unter allen Flüssen $\left(\mathrm{FeNO}_{20-300}\right)$ auf.

Andererseits findet sich für Raucher (im Gegensatz zu Nichtrauchern) keine Korrelation zwischen $\mathrm{D}_{\text {awNo }}$ und $\mathrm{FeNO}_{50-300}$. Eine plausible Erklärung für diese Diskrepanzen zum 2-Kompartimenten-Modell ist, dass in Folge von Turbulenzen und Strömungsinhomogenitäten bei Small airways disease des Rauchers $\mathrm{C}_{\mathrm{alv}}$ und $\mathrm{C}_{\mathrm{aw}}$ nicht separat voneinander ermittelt werden können. Es ist von unterschiedlichen Verzögerungen des expiratorischen Gasabflusses aus dem Alveolarbereich auszugehen. Auch kann ein beträchtlicher Teil des NO aus den Bronchien in den alveolaren Bereich zurückströmen. Dies dürfte dazu führen, dass anstelle von Alveolarluft Mischluft analysiert wird.

Unsere Ergebnisse bezüglich der signifikant erniedrigten $\mathrm{FEF}_{50}$ und $\mathrm{FEF}_{75}$-Werte der Raucher bestätigen (ebenso wie Befunde von anderen Autoren [16-18]) die Annahme, dass die Verengungen der kleinen Bronchien den $\mathrm{C}_{\text {alv }}$-Wert der Raucher beeinflusst. Auch die neuesten Ergebnisse von Mahut et al. [19] bei AsthmaPatienten sprechen dafür, dass erhöhte $\mathrm{C}_{\mathrm{alv}}$-Werte als Indikatoren einer Obstruktion bzw. einer Ventilationsstörung betrachtet werden können. Die Autoren fanden keine Korrelation zwischen dem Asthma-Schweregrad (GINA) und $\mathrm{C}_{\mathrm{alv}}$; demgegenüber bestand 
zwischen den FEF-Werten und $\mathrm{C}_{\mathrm{alv}}$ eine signifikante negative Korrelation.

Eine Vermischung des bronchialen und alveolaren NO wird auch von Hogman et al. [20], Suresh et al. [21] und Shin et al. [22] diskutiert. Hogman et al. [20] und Brindicci et al. [23] fanden signifikant erhöhte $C_{\text {alv }}$-Werte bei Patienten mit COPD, wobei eine Parallelität mit dem klinischen Schweregrad bestand. Letztere Autoren nennen vor allem Inflammationsprozesse in den kleinen Atemwegen als einen möglichen Grund. Hogman et al. [20] gehen außerdem davon aus, dass bei normalem Ventilations-Perfusions-Verhältnis NO während der Inspiration aus den Bronchien zu den Alveolen gelangt und problemlos ins Blut übergeht, während bei Ventilations-Perfusions-Störungen vermehrt nicht diffundiertes NO aus den Bronchien abgeatmet wird. Ob dies in nennenswertem Umfang stattfindet, ist wegen der hohen NODiffusibilität allerdings fraglich. Wir können annehmen, dass Ventilationsstörungen eine wichtigere Rolle als Perfusionsstörungen zukommt.

Es gibt Versuche der Modellierung mit der semi-quantitativen Abschätzung der realen pathologischen Prozesse mit einer differenziellen Betrachtung der Struktur der Atemwege in den verschiedenen Bronchiengenerationen. Mittels Simulation der Bronchienstrukturen nach dem Weibel-Lungenmodell haben Yang et al. [24] theoretisch bewiesen, dass die Obstruktion starke Heterogenitäten der Strömung in den nachfolgenden, sich aufzweigenden Bronchien mit Re-Zirkulationsströmungen verursacht.

Es ist allerdings anzunehmen, dass es sich nicht um eine einfache positive Korrelation zwischen $C_{a l v}$ und $C_{a w}$ bei Rauchern handelt, sondern dass verschiedene Überlagerungen und Interaktionen mit weiteren Effekten wirksam werden, z. B. können die erniedrigten $\mathrm{C}_{\mathrm{aw}}$-Werte mit der Zunahme der Ventilationsstörungen und mit signifikant erhöhten $\mathrm{C}_{\mathrm{alv}}$-Werte einhergehen [23]).

Bemerkenswerterweise werden trotz der vorgenannten Hinweise nur selten in Raucher-Kollektiven signifikant erhöhte $\mathrm{C}_{\text {alv }}$-Werte [23] gefunden, ein Trend zu einer solchen Erhöhung wurde aber von mehreren Untersuchern beobachtet [20,25,26].

\section{Inhomogenitäten vs. axialer Rückströmung}

Es ist zu beachten, dass sich eine NO-Rückströmung in Folge von Strömung-Inhomogenitäten von der einfachen axialen Rückdiffusion (wegen des Gradienten der NO-Konzentration) unterscheidet. Diese Rückdiffusion findet auch in den Atemwegen statt; sie wird allerdings in der klassischen Form des 2-Kompartimenten-Modells vernachlässigt. Die Existenz der NO-Rückströmung in Richtung Alveolen wurde experimentell mittels einer Gasmischung aus Sauerstoff und Helium demonstriert [27].

Eine erweiterte Version des 2-Kompartimenten-Modells von Condorelli et al. [28] (trumpet model with axial diffusion, TMAD) schätzt diesen Effekt der axialen Rückdiffusion ab. Die analytische Lösung dieses Modells ist unter großen Einschränkungen möglich, und zwar unter der linearen Approximation der Gleichung (1), die nur zu den Flüssen $\geq 100 \mathrm{~mL} / \mathrm{s}$ passt (entsprechend zeigen sich hier größere Abweichungen von den realen Daten als im nicht-linearen Modell $[20,25])$. Die lineare Form des TMAD erlaubt keine Abschätzung von $\mathrm{C}_{\mathrm{aw}}$ und $\mathrm{D}_{\mathrm{awNo}}$ und liefert nur Werte für $\mathrm{C}_{\mathrm{alv}}$ und $\mathrm{J}_{\mathrm{aw}}$ (in Form einer Korrektur der Werte des klassischen 2-Kompartimenten-Modells). Diese Korrektur realisiert sich in einem einfachen, 1,7-mal größeren $\mathrm{J}_{\mathrm{aw}}$-Wert für jeden Probanden. Die Korrektur von $\mathrm{C}_{\mathrm{alv}}$ in Folge der Rückdiffusion resultiert in einem niedrigeren Wert und kann zu nicht plausiblen negativen Werten führen (bei Condorelli et al. [28] war $C_{\text {alv }}$ bei 2 von 8 Probanden negativ; in unserer Studie nach Anwendung dieser Korrektur bei 30\% (20 von 67)). Bemerkenswerterweise wurde dieses Problem in den Arbeiten von Gelb et al. [29] und Lehtimaki et al. [30] nicht angesprochen, möglicherweise, weil alle Patienten (Diagnosen: Asthma bzw. COPD) in beiden Studien ursprünglich (vor der Korrektur) relativ hohe $\mathrm{C}_{\mathrm{alv}}$-Werte hatten. In einer Untersuchung von Asthmatikern [31] wurden unter linearer Approximation (ohne Korrektur) negative $C_{\text {alv }}$-Werte für 7 von 26 Patienten erhalten.

Die lineare Form des 2-Kompartimenten-Modells überschätzt den NO-Strom aus der Bronchialwand, sie betrachtet ihn als konstant und unabhängig von der tracheawärts ansteigenden NOKonzentration in den Atemwegen. Das führt zu einer kompensatorischen Unterschätzung (bis hin zu den erwähnten negativen Schätzwerten) der NO-Produktion im alveolo-bronchiolaren Bereich $\left(C_{\text {alv }}\right)$.

Außerdem folgt aus der Korrektur für $\mathrm{C}_{\mathrm{alv}}$, dass zwischen $\mathrm{C}_{\mathrm{alv}}$ und $\mathrm{J}_{\text {aw }}$ des klassischen 2-Kompartimenten-Modells eine positive Korrelation bestehen soll. Wir haben aber eine solche Korrelation weder für Nichtraucher noch für Raucher gefunden.

Es ist auch darauf hinzuweisen, dass der Diffusionsprozess aus der Bronchialwand bei Annahme einer konstanten $C_{a w}$ unabhängig von der Struktur des Bronchialsystems ist [32]. Demgegenüber ist die axiale Diffusion von der Struktur, nämlich vom Querschnitt der Atemwege in den verschiedenen Generationen des Bronchialsystems, abhängig. Die einfache Korrekturformel des TMAD wurde auf Basis der Weibel-Daten [33] für den idealen Querschnitt der Atemwege berechnet. Dabei ist es unklar, wie genau sie Strukturänderungen berücksichtigt. Die axiale Diffusion ist gerade in den peripheren Bronchiengenerationen 12-17 von Bedeutung (großer Querschnitt und große NO-Produktion). Aus pathophysiologischer Sicht bestehen Zweifel, dass in den verengten kleinen Atemwegen bei COPD der NO-Rückfluss in gleichem Umfang wie bei Gesunden stattfindet. Außerdem haben die Computersimulationen des TMAD von Van Muylem et al. [34] gezeigt, dass eine heterogene NO-Produktion (bzw. eine höhere $C_{a w}$ in den Generationen 0 - 3) den Effekt der Rückdiffusion deutlich reduziert.

Das TMAD mit seiner realistischen morphometrischen Struktur kann als ein wichtiger Schritt in die Richtung zu einer realitätsnahen Modellierung des NO-Transports in den Atemwegen betrachtet werden. Andererseits machen die Beschränkungen, unter denen die einfache lineare Lösung des Modells ausgearbeitet wurde, diese Korrekturen fragwürdig.

Differenzielle NO-Parameter, die berechnete Größen darstellen, sind nicht nur von der Berechnungsmethode abhängig, sondern auch von der Wahl der angewandten Flüsse [25,35]. Wir haben gefunden, dass das Ausschließen des niedrigsten Flusses (aus einem Set von 5 Flussraten) zu einer signifikant verminderten Abschätzung von $C_{a w}$ führen kann ([35], Publikation in Vorbereitung). Auch diese Konstellation findet keine Erklärung durch das 2-Kompartimenten-Modell.

Beim Small airways disease mit seinen inflammatorischen Prozessen kommt es zu veränderten Luftströmungen in den peripheren Bronchien. Inhomogenitäten der Struktur des Bronchialbaums realisieren sich in niedrigen Flüssen bei Ausatmung aus schlecht ventilierten Abschnitten (obwohl der gesamte Fluss konstant bleibt) und möglicherweise einer relativ höheren NOProduktion in diesen Bereichen (mit oder ohne Inflammation). Das beeinflusst die exhalierten NO-Konzentrationen und entsprechend die berechneten NO-Parameter. Diese strukturellen Anomalitäten sind bis heute in den verschiedenen Versionen des 2-Kompartimenten-Modells nicht berücksichtigt. 
Wir fanden nur bei Rauchern unter allen Flussraten eine hohe Korrelation zwischen FeNO und $\mathrm{C}_{\mathrm{aw}}$. Wie erwähnt, ist anzunehmen, dass die Abweichung der Daten der Raucher vom 2-Kompartimenten-Modell auf strukturelle Änderungen in Folge der pathophysiologischen Prozesse im alveolo-bronchiolaren Bereich zurückzuführen ist.

Nach traditioneller Ansicht ist die alveolare NO-Konzentration ein Marker der peripheren Atemwegsinflammation, z.B. bei Asthma [30,36] und COPD [23]. Unser Ergebnis bezüglich der starken Korrelation zwischen $\mathrm{C}_{\mathrm{aw}}$ und dem exhalierten NO bei Rauchern relativiert diese Sicht und unterstützt die oben genannte alternative Argumentation von Hogman [20] und Mahut [19] hinsichtlich einer NO-Vermischung.

\section{Auswirkungen des Rauchens}

Rauchen hat verschiedene Wirkungen auf die Bronchialwand und auf den alveolokapillaren Gasaustausch. Die erniedrigte $C_{a w}$ der Raucher ist bedingt durch die biomolekulare Wirkung des Rauchens auf die NO-Synthasen in der Bronchialwand, wahrscheinlich aufgrund der hohen NO-Konzentration im Tabakrauch (Kharitonov et al. [37]). Die Suppression der verschiedenen NOSynthasen in Lungen-Epithel-Zellen und in der glatten Muskulatur von kleinen pulmonalen Gefäßen nach Tabakrauch-Exposition wurde in zahlreichen Studien bewiesen [38-40].

Neben dem Mechanismus der Unterdrückung der NO-Synthase kann es auch zu anderen, nicht so unmittelbaren Wirkungen des Rauchens mit Beeinflussung der NO-Produktion in der Bronchialwand kommen. Rauchen schädigt die Epithelzellen und führt zu inflammatorischen Prozessen mit Up-Regulation verschiedener Enzyme, die NO-Synthasen induzieren können. Das komplizierte Netz der Involvierung von verschiedenen NO-Synthasen demonstriert die neueste Untersuchung von Brindicci et al. [41]. Die Autoren fanden, dass die Aktivitäten der endothelialen NO-Synthase (eNOS) und der induzierten (iNOS) im bronchialen Epithel nicht mit dem Schweregrad der COPD korrelieren, während die Aktivität der neuronalen NO-Synthase (nNOS) in den COPD-Stadien 3-4 nach GOLD signifikant erhöht und mit den Lungenfunktionsparametern $\mathrm{FEV}_{1}$ und $\mathrm{FEV}_{1} / \mathrm{FVC}$ negativ assoziiert ist.

Diese Effekte begleiten die Entwicklung und Exazerbationen der COPD. Sie sind aber unter gesunden Rauchern offensichtlich gering ausgeprägt, da die meisten dieser Probanden eine erniedrigte FeNO (bzw. $\mathrm{C}_{\mathrm{aw}}$ ) aufweisen.

Die vorgenannten experimentellen Befunde lassen unter Rauchern erniedrigte FeNO-Werte erwarten und stimmen gut mit unseren sowie mit den von fast allen anderen Untersuchern festgestellten Ergebnissen überein [42]. Mit dem 2-Kompartimenten-Modell lässt sich die FeNO-Verminderung der Raucher auf die erniedrigten $C_{a w}$-Werte $[20,23,43,44]$ zurückführen. Die vereinzelt abweichenden Ergebnisse mit normaler $C_{a w}$ unter Rauchern sind möglicherweise durch andere Kollektive mit nur geringen pathophysiologischen und morphologischen Veränderungen bedingt [45].

Wir stellen die Hypothese auf, dass die Inhibition der NO-Produktion in der Bronchialwand der Raucher eine Rolle bei der Entwicklung der COPD spielt. NO ist ein Bronchodilatator [46,47]. Es reduziert den Tonus der glatten Bronchialmuskulatur, die die Bronchialwände bis zu den Bronchioli respiratorii umgibt. Die niedrige NO-Konzentration in der Bronchialwand des Rauchers begünstigt demnach eine Verengung der Bronchien, also eine Obstruktion mit konsekutiven pathologischen Prozessen durch Förderung von Schleimretention, Infektionen und chronischen inflammatorischen Prozessen. Dies wirkt sich besonders in den kleinen knorpellosen Bronchien (ab der 11.-12. Bronchiengeneration) aus und könnte erklären, warum die Ventilationsstörung des Rauchers sich vor allem in diesem Atemwegsbereich abspielt. Unsere Ergebnisse - v.a. die erniedrigen FEF-Werte der Raucher und die gefundene positive Assoziation zwischen $\mathrm{FEV}_{1} / \mathrm{FVC}$ und der bronchialen NO-Konzentration $\mathrm{C}_{\mathrm{aw}}$ - sind mit dieser Hypothese einer initialen pathophysiologischen Rolle der verminderten NO-Konzentration in der Bronchialwand gut vereinbar.

\section{Interessenkonflikt}

$\nabla$

Die Autoren geben an, dass kein Interessenkonflikt besteht.

\section{Literatur}

1 Kharitonov SA, Yates D, Robbins RA et al. Increased nitric oxide in exhaled air of asthmatic patients. Lancet 1994; 343: $133-135$

2 Silvestri M, Spallarossa D, Frangova Yourukova $V$ et al. Orally exhaled nitric oxide levels are related to the degree of blood eosinophilia in atopic children with mild-intermittent asthma. Eur Respir J 1999; 13: $321-326$

3 Ihre E, Gyllfors P, Gustafsson LE et al. Early rise in exhaled nitric oxide and mast cell activation in repeated low-dose allergen challenge. Eur Respir J 2006; 27: 1152 - 1159

4 Spanier AJ, Kahn RS, Hornung RW et al. Environmental exposures, nitric oxide synthase genes, and exhaled nitric oxide in asthmatic children. Pediatr Pulmonol 2009; 44: 812-819

5 Smith $A D$, Cowan JO, Filsell $S$ et al. Diagnosing asthma. Comparisons between exhaled nitric oxide measurements and conventional tests. Am J Respir Crit Care Med 2004; 169: 473-478

6 Zacharasiewicz A, Wilson N, Lex C et al. Clinical use of non-invasive measurements of airway inflammation in steroid reduction in children. Am J Respir Crit Care Med 2005; 171: 1077 - 1082

7 Zeiger RS, Szefler SJ, Phillips BR et al. Response profiles to fluticasone and montelukast in mild-to-moderate persistent childhood asthma. J Allergy Clin Immunol 2006; 117: 45-52

8 Tsujino I, Nishimura M, Kamachi A et al. Exhaled nitric oxide - is it really a good marker of airway inflammation in bronchial asthma? Respiration 2000; 67: 645-651

9 Tsoukias NM, George SC. A two-compartment model of pulmonary nitric oxide exchange dynamics. J Appl Physiol 1998; 85: 653-666

10 Silkoff PE, Sylvester JT, Zamel $N$ et al. Airway nitric oxide diffusion in asthma: role in pulmonaly function and bronchial responsiveness. Am J Respir Crit Care Med 2000; 161: 1218-1228

11 Silkoff PE, McClean PA, Slutzky AS et al. Marked flow-dependence of exhaled nitric oxide using a new technique to exclude nasal nitric oxide. Am J Respir Crit Care Med 1997; 155: 260 - 267

12 Lehtimaki L, Kankaanranta $H$, Saarelainen $S$ et al. Inhaled fluticasone decreases bronchial but not alveolar nitric oxide output in asthma. Eur Respir J 2001; 18: 635-639

13 American Thoracic Society. ATS/ERS Recommendations for standardized procedures for the online and offline measurement of exhaled lower respiratory nitric oxide and nasal nitric oxide, 2005. Am J Respir Crit Care Med 2005; 171: $912-930$

14 Baur X, Barbinova L. Latex allergen exposure increases exhaled nitric oxide in symptomatic health care workers. Eur Respir J 2005; 25: 309-316

15 Jörres RA. Modelling the production of nitric oxide within the human airways. Eur Respir j 2000; 16: 555 - 560

16 Gold DR, Wang X, Wypij D et al. Effects of cigarette smoking on lung function in adolescent boys and girls. N Engl J Med 1996; 335: 931 937

17 Buist AS, Vollmer WM, Wu Y et al. Effects of cigarette smoking on lung function in four population samples in the People's Republic China. The PRS-US Cardiovascular and Cardiopulmonary Research Group. Am J Respir Crit Care Med 1995; 151: 1393 - 1400

18 Milaat WA, el-Ganai FM. Effects of cigarette smoking on lung function of Saudi students. Asia Pac J Public Health 1998; 10: 39-42

19 Mahut B, Trinquart L, Le Bourgeois $M$ et al. Multicentre trial evaluating alveolar NO fraction as a marker of asthma control and severity. Allergy 2009; [Epub ahead of print] 
20 Hogman M, Holmkvist, Wegener T et al. Extended NO analysis applied to patients with COPD, allergic asthma and allergic rhinitis. Respir Med 2002; 96: $24-30$

21 Suresh $V$, Shelley $D A$, Shin $H W$ et al. Effect of heterogeneous ventilation and nitric oxide production on exhaled nitric oxide profiles. J Appl Physiol 2008; 104: 1743-1752

22 Shin HW, Condorelli P, Rose-Gottron CM et al. Probing the impact of axial diffusion on nitric oxide exchange dynamics with heliox. Appl Physiol 2007; 97: 874-882

23 Brindicci $C$, Ito $K$, Resta $O$ et al. Exhaled nitric oxide from lung periphery is increased in COPD. Eur Respir J 2005; 26: 52 - 59

24 Yang XL, Liu Y, Luo HY. Respiratory flow in obstructed airways. J Biomech 2006; 39: $2743-2751$

25 Roy K, Borrill ZL, Starkey C et al. Use of different exhaled nitric oxide multiple flow rate models in COPD. Eur Respir J 2007; 29: 651 - 659

26 Brindicci $C$, Ito $K$, Torre $O$ et al. Effects of aminoguanidine, an inhibitor of inducible nitric oxide synthase, on nitric oxide production and its metabolites in healthy control subjects, healthy smokers and COPD patients. Chest 2009; 135: $353-367$

27 Shin HW, Condorelli P, Rose-Gottron CM et al. Probing of impact of axial diffusion on nitric oxide exchange dynamics with heliox. J Appl Physiol 2004; 97: 874-882

28 Condorelli P, Shin HW, Aledia AS et al. A simple technique to characterize proximal and peripheral nitric oxide exchange using constant flow exhalations and an axial diffusion model. J Appl Physiol 2007; 102: $417-25$

29 Gelb AF, Flynn Taylor CF, Krishnan A et al. Central and peripheral airways sites of nitric oxide gas exchange in COPD. Chest 2010; 137: $575-84$

30 Lehtimaki L, Kankaanranta H, Saarelainen S et al. Inhaled fluticasone decreases bronchial nitric oxide is related to symptom relief during fluticasone treatment in COPD. Eur Respir J 2010; 35: 72 - 78

31 van Veen IH, Sterk PJ, Schot $R$ et al. Alveolar nitric oxide versus measures of peripheral airway dysfunction in severe asthma. Eur Respir J 2006; 27: 951 - 956

32 Barbinova L, Baur X. Weibel's Modell der Morphometrie der Lungen (WML) und die Voraussetzungen des 2-Kompartimenten Modell (2KM) der NO-Produktion. Pneumologie 2010; 64: S176

33 Weibel ER. Morphometry of the human lung. Berlin: Springer-Verlag; 1963
34 Van Muylem A, Noel C, Paiva M. Modelling of impact of gas molecular diffusion on nitric oxide expired profile. J Appl Physiol 2003; 94: $119-127$

35 Barbinova L, Baur X. Effects of flow rates on the estimation of differential NO Parameters. Am J Respir Crit Care Med 2010; 181: A4270

36 Gelb AF, Taylor CF, Nussbaum E et al. Alveolar and airway sites of nitric oxide inflammation in treated asthma. Am J Respir Crit Care Med 2004; $170: 737-741$

37 Kharitonov SA, Robbins RA, Yates D et al. Acute and chronic effects of cigarette smoking on exhaled nitric oxide. Am J Respir Crit Care Med 1995; 152: 609-612

38 Hoyt JC, Robbins RA, Habib M et al. Cigarette smoke decreases inducible nitric oxide synthase in lung epithelial cells. Exp Lung Res 2003; 29: $17-28$

39 Rytila P, Rehn T, Ilumets $H$ et al. Increased oxidative stress in asymptomatic current chronic smokers and GOLD stage 0 COPD. Respir Res 2006; 7: 69

40 Ferrer E, Peinado VI, Diez M et al. Effects of cigarette smoke on endothelial function of pulmonary arteries in the guinea pig. Respir Res 2009; 10: 76

41 Brindicci $C$, Kharitonov SA, Ito $M$ et al. Nitric oxide synthase isoenzyme expression and activity in peripheral lung tissue of patients with chronic obstructive pulmonary disease. Am J Respir Crit Care Med 2010; $181: 21-30$

42 Malinovschi A, Janson C, Holmkvist T et al. Effect of smoking on exhaled nitric oxide and flow-independent nitric oxide exchange parameters. Eur Respir J 2006; 28: 339- 345

43 Pietropaoli AP, Perillo IB, Perkins PT et al. Smokers have reduced nitric oxide production by conducting airways but normal levels in the alveoli. Inhal Toxicol 2007; 19: 533-541

44 Olin AC, Rosengren A, Thelle DS et al. Height, age, and atopy are associated with fraction of exhaled nitric oxide in a large adult general population sample. Chest 2006; 130: 1319-1325

45 Borrill ZL, Roy K, Vesey RS et al. Non-invasive biomarkers and pulmonary function in smokers. Inter J Chron Obstruct Pulmon Dis 2008; 3 : $171-183$

46 Dupuy PM, Shore SA, Drazen JM et al. Bronchodilator action of inhaled nitric oxide in guinea pigs. Clin Invest 1992; 90: 421 - 428

47 Sanna A, Kurtansky A, Veriter $C$ et al. Bronchodilator effect of inhaled nitric oxide in healthy men. Am J Respir Crit Care Med 1994; 150 $1702-1704$ 